back pain (IBP) by ASAS criteria. The examination included X-ray of sacroiliac joints (SIJs) (pelvic radiographs), HLA B27 antigen status; magnetic resonance imaging (MRI) of SIJs was performed in 79 pts, regardless of the presence of IBP, on Signa Ovation 0,35T. Bone marrow oedema on MRI (STIR) was considered as active MRI sacroiliitis (MRI-SI). Radiographic sacroiliitis (R-SI) was considered according to New York criteria (unilateral grade $\geq 3$ or bilateral grade $\geq 2$ ). X-ray and MRI results were evaluated by an independent reader. IBP was observed in 63 cases (66.3\%), MRI-SI in 28 of 79 (35.4\%) examined cases, R-SI in 29 cases $(30.5 \%)$. Pts were split into two groups (gr.): those with axial involvement (axPsA), that is with IBP and/or R-SI and/or MRI-SI; and those without axial involvement (having only peripheral PsA [pPsA]). The axPsA gr. included $65(68.4 \%)$ cases, the pPsA gr. $-30(31.6 \%)$ cases. Skin lesion severity was evaluated in terms of body surface area (BSA) affected and Psoriasis Area Severity Index (PASI). When BSA was $\geq 3 \%$, PASI was calculated. PASI $\geq 11$ indicates moderate and severe psoriasis.

Results: The following significant differences were revealed between gr. axPsA and gr. pPsA: gender-related ones: in gr. axPsA men-to-women ratio was $60.0 \%$ to $40.0 \%$, while in gr. pPsA it was $26.7 \%$ to $73.3 \%$ ( $p=0.003$ ); age-related ones: mean age of. gr. axPsA was $33.9 \pm 9.6$ years, while of gr. pPsA it was 41.7 \pm 10.6 years $(p=0.0007)$; in disease duration: in gr. axPsA it was $10.3 \pm 8.7 \mathrm{mo}$. which was less than gr. pPsA $16.1 \pm 11.7$ mo. ( $p=0.008)$; in HLA-B27 antigen status: in gr. axPsA it was positive in $47.6 \%$ of pts. while in gr. pPsA in $23.3 \%$ of pts. $(\mathrm{p}=0.02)$; in PGA: $58.4 \pm 17.3 \mathrm{~mm}$ in gr. axPsA, and $49.8 \pm 16.7 \mathrm{~mm}$ in gr. pPsA $(\mathrm{p}=0.02)$; in skin lesions' severity: it was higher in gr. axPsA: BSA median was 3.0 [1.0-9.0] in gr. axPsA and $1.0[0.2-3.0]$ in gr. pPsA $(p=0.007)$, and PASI median was $15.6[6.6-55.2]$ in gr. axPsA and 6.0 [0.0-7.2] in gr. pPsA $(p=0.006)$.

Conclusions: Axial involvement in early PsA patients is significantly more frequent in males and in pts with positive HLA-B27 antigen status. Axial involvement is associated with high patient-reported disease activity and severe skin lesion. These findings may have a positive impact on diagnosis of axial disease, as well as on the selection of the best therapeutic strategy.

Disclosure of Interest: None declared

DOI: 10.1136/annrheumdis-2018-eular.2385

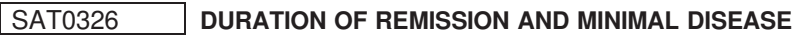 ACTIVITYAFTER STARTING AND DISCONTINUATION OF BIOLOGIC (B) DMARDS IN EARLY PSORIATIC ARTHRITIS PATIENTS TREATED ACCORDING TO TREAT-TO-TARGET STRATEGY (RESULTS OF AN OPEN-LABEL REMARCA STUDY)}

E. Loginova, T. Korotaeva, E. Gubar, S. Glukhova, E. Nasonov. Nasonova Research Institute of Rheumatology, Moscow, Russian Federation

Background: The main goal of treat-to-target (T2T) strategy in psoriatic arthritis (PsA) is achievement of remission (REM) or minimal disease activity (MDA). There is limited data concerning the duration of REM/MDA after starting and discontinuation of bDMARDs in early (E) PsA patients (pts).

Objectives: to investigate the timing and duration of REM/MDA after starting and discontinuation of bDMARDs therapy in EPsA pts treated according to T2T strategy.

Methods: 34 (M-18/F-16) pts with active EPsA according to the CASPAR criteria non-responder to methotrexate (MTX) subcutaneous treatment, bDMARD-naïve were included; mean age $38 \pm 11$ years, PsA duration $12 \pm 10$ months (mon.), psoriasis duration 89.8 \pm 91.1 mon., median Disease Activity index for Psoriatic Arthritis (DAPSA) 33.55 [28.34-41.77]. All pts started bDMARDs: Adalimumab (21 pts), Certolisumab pegol (3 pts), Etanercept (2 pts), Ustekinumab (8 pts) \pm MTX. The median duration of bDMARDs treatment was 9 [6.5-15], Min 3 mon. - Max 24 mon. The bDMARDs treatment was stopped due to an individual inefficiency, loss of efficacy or non-medical reasons. At baseline and every 3 mon. all pts underwent assessment of PSA activity by DAPSA and MDA criteria (tender joint count $\leq 1$, swollen joint count $\leq 1, \mathrm{PASI} \leq 1$ or $\mathrm{BSA} \leq 3$, patient pain global assessment $V A S \leq 15$, patient's global disease activity $V A S \leq 20, H A Q \leq 0,5$, enthesitis count $\leq 1$ ). The proportion of pts who achieved REM by DAPSA $\leq 4$ and MDA ( 5 of 7 cutpoints) at least once, as well as timing and duration of REM/MDA after starting and cessation of bDMARDs were performed. Pts reports about a flare coming, close to the scheduled time of assessment, was taken into account. $M \pm S D, M e$ [Q75; Q50], (\%) were calculated. All $p<0.05$ were considered to indicate statistical significance.

Results: By the 24 mon. of the study REM by DAPSA and MDA was reached at least once by $27(79 \%)$ and $28(82 \%)$ out of 34 pts, accordingly. Mean timing of REM by DAPSA and MDA after starting of bDMARDs was $5.8 \pm 3.2$ and 4.0 \pm 1.9 mon., accordingly. During the observation 19 out of 27 pts $(70.4 \%)$ had stopped bDMARDs due to different reasons. 8 out of 27 pts (29.6\%) continued the treatment and had REM according to DAPSA by the end of the study. After cessation of bDMARDs flares by DAPSA were seen in 12 out of 19 pts $(63.2 \%)$ with the mean duration of REM 5.8 \pm 2.3 mon. (Fig 1.). The loss of MDA was seen in 12 out of 20 pts $(60 \%)$ with the mean duration of MDA $6.2 \pm 3.0$ mon. Pts reports about the time of PsA symptoms flares was $3.5 \pm 3.4$ mon. 5 out of 34 pts $(15 \%)$ loss the efficacy after $12.0 \pm 4.7$ mon. of bDMARDs treatment.

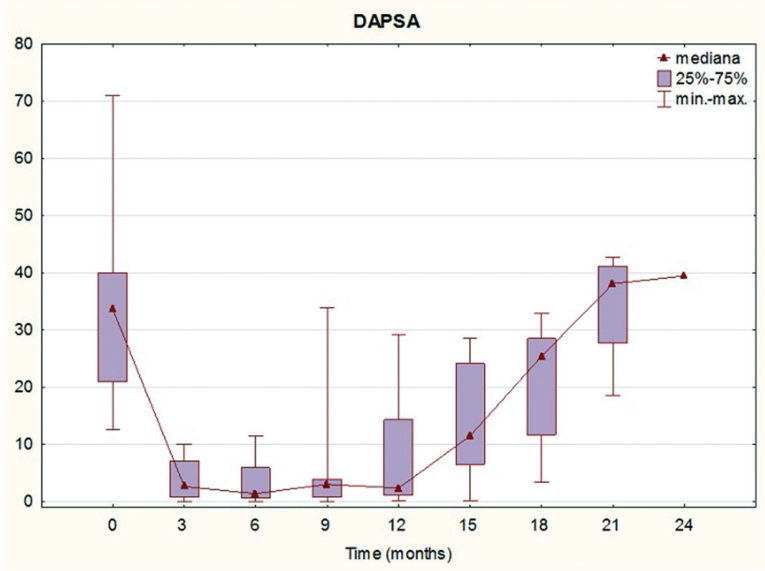

Abstract SAT0326 - Figure 1. Dynamics of DAPSA after starting and discontinuation of bDMARDs therapy in EPsA.

Conclusions: Most EPsA pts treated with bDMARDs according to T2T strategy achieved REM and MDA within 5 mon. Flares after bDMARDs discontinuation within 6 mon were found in more than a half of pts. according to PsA activity indices and within 3 mon. by pts reports. REM and flare timing after bDMARDs therapy discontinuation indicate that further studies concerning optimal management PsA pts by bDMARDs are needed.

Disclosure of Interest: None declared

DOI: 10.1136/annrheumdis-2018-eular.3245

\section{SAT0327 BASELINE CHARACTERISTICS AND REASONS FOR APREMILAST PRESCRIPTION IN A LARGE ITALIAN COHORT OF PSORIATIC ARTHRITIS PATIENTS}

E.G. Favalli ${ }^{1}$, C. Zuccaro ${ }^{2}$, M. Biggioggero ${ }^{1}$, C. Selmi ${ }^{3}$, R. Bucci ${ }^{4}$, F. D'onofrio ${ }^{5}$, G. Carlino ${ }^{6}$, L. Santo ${ }^{7}$, A. Semeraro ${ }^{8}$, S. Monti ${ }^{9}$, D. Mazzotta ${ }^{10}$, F. Atzeni $^{11}$,

R. Caporali ${ }^{9}, \mathrm{~F}$. lannone ${ }^{12}$. ${ }^{1}$ Department of Rheumatology, Gaetano Pini Institute, Milano; ${ }^{2}$ Hospital Outpatient clinic of Rheumatology, ASL BR, Brindisi;

${ }^{3}$ Rheumatology, Humanitas Research Hospital, BIOMETRA Department, University of Milan, Milano; ${ }^{4}$ Rheumatology Hospital Unit, A.O.U. "OO.RR. Foggia",

${ }^{5}$ UOC Reumatologia Universitaria, University of Foggia, Foggia; ${ }^{6}$ Rheumatology Service, ASL Lecce - DSS Casarano and Gallipoli, Lecce; ${ }^{7}$ Rheumatology Service, DSS4 Barletta ASL BT Andria, Andria; ${ }^{8}$ UO of Rheumatology, ASL Taranto, Taranto; ${ }^{9}$ Department of Rheumatology, University of Pavia, IRCCS Policlinico San Matteo Foundation, Pavia; ${ }^{10}$ Hospital Outpatient clinic of Rheumatology, ASL BR, Brindisi, Brindisi; ${ }^{11}$ Rheumatology, University of Messina, Messina; ${ }^{12}$ DETO-Rheumatology Unit, University of Bari, Bari, Italy

Background: There are no real-world data for the profiling of patients with psoriatic arthritis (PsA) receiving the phosphodiesterase-4 inhibitor apremilast. Objectives: To retrospectively evaluate the baseline characteristics and the reasons for apremilast prescription in a large Italian multicenter cohort of PsA cases (Real-life APremilast for Psoriatic arthritis Evaluation Registry, RAPPER).

Methods: Data were retrospectively extracted from the RAPPER registry which includes all PsA cases treated with apremilast in 11 Italian tertiary rheumatology centres between January 2017 and December 2017. Descriptive analysis of baseline characteristics of study population included demographics, previous treatments before apremilast, pattern of PsA involvement, disease activity indices, and prevalence of comorbidities (computed by the Rheumatic Disease Comorbid ity Index [RDCI $\left.{ }^{1}\right)$. Reasons for apremilast choice were also analysed.

Results: We studied 97 patients with PsA (61\% women; mean [ \pm standard deviation, SD] age $56.7 \pm 11.9$ years; mean disease duration $10 \pm 13.1$ years) who received apremilast as first-line targeted disease modifying drug $(51.5 \%)$ or after the failure of at least one biologic agent $(48.5 \%)$. In $75 \%, 33 \%, 11 \%$, and $63 \%$ of patients there were articular (57\% asymmetric oligoarthritis; mean Disease Activity in PSoriatic Arthritis [DAPSA] 22.55 \pm 11.55 ), entheseal (mean Leeds Enthesitis Index [LEI] 2.63 \pm 1.42 ), axial (mean Bath Ankylosing Spondylitis Disease Activity Index [BASDAI] 6.02 \pm 2.40 ), and skin/ungueal (mean body surface area [BSA] $1.71 \pm 3.44)$ involvement, respectively. Two thirds $(64 \%)$ of patients had at least one comorbidity (mean $\mathrm{RDCl} 1.20$ ) and the prevalence of conditions is reported in table 1. The main reasons for apremilast prescription were contraindication to biologic agents (66\%), lack of poor prognostic factors (35\%), comorbidities (34\%), 
risk of infections (33\%), and history of malignancy (22.6\%), whereas a preference for an oral drug drove the choice only in $7 \%$ of patients.

Abstract SAT0327 - Table 1. baseline prevalence of comorbidities

\begin{tabular}{|l|c|}
\hline COMORBIDITY & PREVALENCE $(\mathrm{n}=97)$ \\
\hline Hypertension & $34 \%$ \\
\hline History of malignancy & $22 \%$ \\
\hline Fibromyalgia & $15 \%$ \\
\hline Latent tuberculosis & $14 \%$ \\
\hline Liver disease & $14 \%$ \\
\hline Lung disease & $13 \%$ \\
\hline Dyslipidemia & $12 \%$ \\
\hline Diabetes & $11 \%$ \\
\hline Depression & $10 \%$ \\
\hline HBV/HCV infection & $9 \%$ \\
\hline Osteoporosis & $8 \%$ \\
\hline Hematological disorders & $7 \%$ \\
\hline Cardiovascular disease & $7 \%$ \\
\hline Gastrointestinal disorders & $3 \%$ \\
\hline Neurological disorders & $3 \%$ \\
\hline
\end{tabular}

Conclusions: Based on our analysis, apremilast is mainly used in PsA with oligoarthritis, enthesitis, mild skin involvement, and low risk of disease progression, carrying comorbidities (especially history of infections and malignancies) with contraindications to the use of biologic drugs.

\section{REFERENCE:}

[1] England BR, Sayles $H$, Mikuls TR, et al. Validation of the rheumatic disease comorbidity index. Arthritis Care Res 2015;67(6):865-72.

Disclosure of Interest: None declared DOI: 10.1136/annrheumdis-2018-eular.4139

\section{SAT0328 WEIGHT-LOSS IMPROVES DISEASE ACTIVITY IN OBESE PATIENTS WITH PSORIATIC ARTHRITIS}

E. Klingberg ${ }^{1}$, A. Bilberg ${ }^{2}$, S. Björkman ${ }^{3}$, B. Eliasson ${ }^{4}$, I. Larsson ${ }^{3} .{ }^{1}$ Department of Rheumatology and Inflammation Research, SAHLGRENSKA ACADEMY AT UNIVERSITY OF GOTHENBURG, Göteborg; ${ }^{2}$ Institute of Neuroscience and Physiology, Section of Health and Rehabilitation, Physiotherapy, Sahlgrenska Academy at the University of Gothenburg; ${ }^{3}$ Department of Gastroenterology and Hepatology; ${ }^{4}$ Department of Endocrinology, Diabetology and Metabolism, Sahlgrenska University Hospital, Gothenburg, Sweden

Background: Obesity is over-represented in patients with psoriatic arthritis (PsA) and associated with increased risk of getting the disease, higher disease activity, poorer effect of treatment and in addition cardiovascular co-morbidity.

Objectives: The aim of this study was to determine the effects of weight-loss treatment on disease activity in patients with PsA and obesity

Methods: Patients with PsA (Caspar criteria) and obesity (body mass index $\mathrm{BMl}>33 \mathrm{~kg} / \mathrm{m}^{2}$ ) where recruited from three Rheumatology clinics in the Western Sweden. Weight-loss treatment with Very Low Energy liquid Diet (VLED), which comprises a daily energy intake of $640 \mathrm{kcal}$, was given during 12-16 weeks, depending on BMI. The first strict period was followed by a structured reintroduction of an energy restricted diet during 18 weeks. The treatment was given within the framework of a structured program including support and medical follow-up at the Obesity Unit at Sahlgrenska University Hospital.

Treatment with DMARDs and biologics was held constant from 3 month before baseline until 6 months after baseline. The patients were assessed with 66/68 joints count, back-mobility tests, body surface area (BSA), questionnaires, ESR, $\mathrm{CRP}$ and $\mathrm{BMI}$ at baseline, 3 and 6 months. The number of patients reaching Psoriatic Arthritis Response Criteria (PsARC) and Minimal Disease Activity (MDA) was calculated.

Results: Totally 41 PsA patients, median age 54 (IQR 48-62) years, 63\% women were included. The median weight-loss was 18.7 (IQR 14.6-26.5) kg. BMI decreased from median $35.2(34.1-38.1)$ to $29.7(26.2-31.5) \mathrm{kg} / \mathrm{m}^{2}$. A majority of the disease activity parameters improved significantly (Table). Totally 16 patients $(39.0 \%)$ reached PsARC and the number of patients with MDA increased from $n=12(29 \%)$ to $n=22(54 \%), p<0.001$ between baseline and the 6 months visit. Conclusions: Weight-loss treatment with VLED had significant positive effects on disease activity in joints, entheses and skin in patients with PsA and obesity. Disclosure of Interest: E. Klingberg Grant/research support from: The VLED used in this study was sponsored by Cambridge Weight Plan., A. Bilberg: None declared, S. Björkman: None declared, B. Eliasson: None declared, I. Larsson: None declared

\begin{tabular}{lccc}
\hline $\mathbf{n = 4 1}$ & $\begin{array}{c}\text { BASELINE } \\
\text { Median (IQR) }\end{array}$ & $\begin{array}{c}\text { 6 MONTHS } \\
\text { Median (IQR) }\end{array}$ & p-value \\
\hline Tender joints 68, score & $4(1-14)$ & $2(0-6.5)$ & $<0.001$ \\
Swollen joints 66, score & $0(0-1)$ & $0(0-0.5)$ & $\mathbf{0 . 0 2 1}$ \\
Leeds enthesitis index & $2(0-4)$ & $0(0-3)$ & $\mathbf{0 . 0 0 1}$ \\
BSA,\% & $0,75(0-2.25)$ & $0.25(0-1.25)$ & $\mathbf{0 . 0 1 4}$ \\
BASMI, score & $2.8(1.8-3.6)$ & $2.8(2.0-3.4)$ & $\mathbf{0 . 6 6 4}$ \\
CRP & $4.0(2.0-8.5)$ & $2.0(1.0-6.5)$ & $\mathbf{0 . 0 4 1}$ \\
ESR, mm/h & $12.0(5.5-18.0)$ & $14(6.0-27.5)$ & $\mathbf{0 . 0 0 8}$ \\
VAS Pain, mm & $30(17-62)$ & $20(5.0-56.5)$ & $\mathbf{0 . 0 0 5}$ \\
VAS Global health, mm & $33(17.5-57.3)$ & $14(6-51)$ & $\mathbf{0 . 0 0 2}$ \\
VAS Fatigue, mm & $55(21-66)$ & $30(8-44)$ & $\mathbf{0 . 0 0 1}$ \\
HAQ, score & $0.63(0.13-1.0)$ & $0.25(0-0.63)$ & $<\mathbf{0 . 0 0 1}$ \\
DAS-28, score & $2.62(1.92-4.02)$ & $2.44(1.97-3.69)$ & $\mathbf{0 . 0 1 3}$ \\
\hline
\end{tabular}

DOI: 10.1136/annrheumdis-2018-eular.4235

\section{SAT0329 FIBROMYALGIA IN PATIENT WITH PSORIATIC ARTHRITIS: RELATIONSHIP WITH ENTHESOPATHY, SLEEP, FATIGUE AND QUALITY OF LIFE}

F. Ulutatar ${ }^{1}$, C. Unal ${ }^{1}$, M.T. Duruoz ${ }^{2} .{ }^{1} P M R$ Department, ${ }^{2} P M R$ Department, Rheumatology Division, Marmara University School of Medicine, Istanbul, Turkey

Background: Fibromyalgia is a syndrome characterised by musculoskeletal pain, sleep disturbance and fatigue. Fibromyalgia commonly accompanies rheumatic diseases. Enthesopathy seen in psoriatic arthritis (PsA) patients may have effect on fibromyalgia.

Objectives: To evaluate relationship of fibromyalgia with enthesopathy, sleep, fatigue and quality of life in patients with psoriatic arthritis.

Methods: The psoriatic arthritis patients according to CASPAR criteria were included into the study. Fibromyalgia diagnosis was based on 2010 ACR criteria. Demographic (age, gender, marital status, education, body mass index) and clinical parameters (disease duration, comorbidities, Moll-white classification of PsA) were noted. Disease activity and enthesopathy were evaluated with Disease Activity Score-28 (DAS-28) and Maastricht Ankylosing Spondylitis Enthesitis Score (MASES), respectively. Functional assessment scales in this study were Psoriatic Arthritis Quality of Life (PsAQoL), Pittsburgh Sleep Quality Index (PSQI), Multidimensional Assessment of Fatigue (MAF). Fibromyalgia Impact Questionnaire (FIQ) was used to assess functional status of fibromyalgia. The difference in mean scores between fibromyalgia and the patients without fibromyalgia were analysed with Mann-Whitney U test. Spearman correlation coefficient (rho) was used for correlations between functional parameters. $p<0.05$ was accepted as significant.

Results: We enrolled 50 PsA patients (32 female, 18 male) with a mean age 49.6 years (SD: 10.4) and mean disease duration 90.2 months (SD: 90.8). Thirty-two patients ( $64 \%$ of PsA patients) fulfilled ACR criteria for fibromyalgia. The mean scores of MASES, PSQI, MAF and PSAQoL were significantly higher in patients with fibromyalgia $(\mathrm{p}<0.05)$. The correlations between FIQ and other functional parameters were as follows; MASES (rho $=0.73, \mathrm{p}<0.0005$ ), PSQI (rho $=0.56$ $\mathrm{p}<0.0005)$, MAF (rho=0.63, $\mathrm{p}<0.0005)$, PsAQoL (rho=0.69, $\mathrm{p}<0.0005)$. There was no significant correlation between FIQ and disease duration $(p>0.05)$. Weak correlation was existing between FIQ and DAS-28 ( $r h o=0.30, p=0.03$ ).

Abstract SAT0329 - Table 1. Clinical characteristics of patients $(n=50)$

\begin{tabular}{lccc}
\hline & Min-Max & $\begin{array}{c}\text { Median } \\
\text { (SD) }\end{array}$ & $\begin{array}{c}\text { Lower-Upper } \\
\text { Quartiles }\end{array}$ \\
\hline DAS-28 & $1,20-$ & $2,60^{1,18}$ & $1,99-3,50$ \\
MASES & 5,60 & & \\
PSQI & $0-13$ & $3,00^{4,84}$ & $0,00-10,00$ \\
PSAQOL & $0-14$ & $6,00^{4,50}$ & $2,00-11,00$ \\
MAF & $0-20$ & $6,00^{5,79}$ & $1,75-11,25$ \\
FIQ & $1-45,80$ & $27,25^{14,98}$ & $10,75-37,75$ \\
\hline DAS-28: & $0-92,82$ & $32,25^{26,54}$ & $10,75-52,73$ \\
\hline
\end{tabular}

DAS-28: Disease activity score-28, MASES: Maastricht Ankylosing Spondylitis Enthesitis Score, PSQI: Pittsburgh Sleep Quality Index, PSAQOL: Psoriatic Arthritis Quality of Life, MAF: Multidimensional Assessment of Fatigue, FIQ: Fibromyalgia Impact Questionnaire, SD: Standart Deviation

Conclusions: Coexistence of fibromyalgia in PsA patients is associated with presence of enthesopathy, poor quality of life, sleep disturbance and fatigue. It is an important aspect to diagnose and treat fibromyalgia in PsA patients for comprehensive treatment. 